\title{
On explicit strong solution of Itô-SDE's and the Donsker delta function of a diffusion
}

\author{
Alberto Lanconelli ${ }^{1), 2)}$ and Frank Proske ${ }^{2)}$
}

July 16, 2003

1) Department of Mathematics, University of Pavia, via Ferrata,1 , 27100 Pavia, Italy

2) Department of Mathematics, University of Oslo

Box 1053 Blindern, N-0316 Oslo, Norway

e-mails: albertol@math.uio.no,proske@math.uio.no

\begin{abstract}
We determine a new explicit representation of the strong solution of Itô-diffusions and elicit its correspondence to the general stochastic transport equation. We apply this formula to deduce an explicit Donsker delta function of a diffusion.
\end{abstract}

Key words and phrases: Itô-diffusions, SPDE, white noise analysis, Donsker delta function, Malliavin calculus, stochastic flows

AMS 1991 classification: 60H15; 60H40

\section{Introduction}

One of the main objectives of this paper is to derive an explicit formula for global strong solutions of the Itô-diffusion

$$
d X_{t}^{x}=b\left(t, X_{t}^{x}\right) d t+\sigma\left(t, X_{t}^{x}\right) d B_{t}, X_{0}=x,
$$

where $B_{t}$ is the Brownian motion. We show that the strong solution of (1.1) corresponds to a solution of a general stochastic transport equation driven by Gaussian white noise (see [7]), which comprises the associated stochastic flow equation(see [9]) as a special case. As an application of this latter result we establish under certain conditions a closed formula for the Donsker delta function of a diffusion. Several researchers investigated solutions of (1.1) and obtained closed form solutions. In [8] and [20] the authors find explicit formulas in terms of the Itô-Wiener chaos expansion. See also [15]. Another essential contribution to solutions of SDE's can be found in [9], where techniques of the theory of stochastic flows are invoked. Our representation of the solution is different from the above mentioned ones and we believe that it conveys new insight into the structure of the solution from a different point of view. Our method is based upon white noise analysis concepts. 


\section{Framework}

In this Section we give a short review of concepts of white noise analysis which we utilize later on to determine an explicit formula for strong solutions and the Donsker delta function of the diffusion (1.1). For background information in white noise analysis we recommend the reader to consult the nice books [6], [12], [17], [7]. In this paper we adopt the notation of the book [7]. ¿From now on we assume that our Brownian motion is constructed on the white noise probalility space

$$
(\Omega, \mathcal{A}, \mathcal{P})=\left(S^{\prime}(\mathbb{R}), \mathcal{B}\left(S^{\prime}(\mathbb{R})\right), \mu\right),
$$

where $S^{\prime}(\mathbb{R})$ is the Schwartz distribution space, $\mathcal{B}\left(S^{\prime}(\mathbb{R})\right)$ the Borel topology on $S^{\prime}(\mathbb{R})$ and $\mu$ the white noise measure. Further we indicate by $(S)$ respectively $(S)^{*}$ the Hida test function space and the Hida distribution space, using the orthonormal basis for $\mathcal{L}^{2}(\mathbb{R})$ consisting of the Hermite functions $\left(\xi_{k}\right)_{k \geq 1}$, one can define an orthogonal $\mathcal{L}^{2}(\mu)$ basis

$$
\left\{\mathcal{H}_{\alpha}(\omega)\right\}_{\alpha \in \mathcal{I}},
$$

where $\mathcal{I}$ stands for the collection of all multi-indices $\alpha=\left(\alpha_{1}, \alpha_{2}, \ldots\right)$ with finitely many non zero entries $\alpha_{i} \in \mathbb{N}_{0}$. So every $F \in \mathcal{L}^{2}(\mu)$ can be uniquely written as

$$
F(\omega)=\sum_{\alpha} c_{\alpha} \mathcal{H}_{\alpha}(\omega) ; c_{\alpha} \in \mathbb{R}
$$

with isometry

$$
\|F\|_{\mathcal{L}^{2}(\mu)}^{2}=\sum_{\alpha} \alpha ! c_{\alpha}^{2}
$$

where $\alpha !=\alpha_{1} ! \alpha_{2} ! \cdots$ for $\alpha=\left(\alpha_{1}, \alpha_{2}, \ldots\right) \in \mathcal{I}$. Then the Hida test function space $(S)$ can be characterized as the collection of all $f(\omega)=\sum_{\alpha} c_{\alpha} \mathcal{H}_{\alpha}(\omega) \in \mathcal{L}^{2}(\mu)$ such that

$$
\|f\|_{0, q}^{2}:=\sum_{\alpha} \alpha ! c_{\alpha}^{2}(2 \mathbb{N})^{q \alpha}<\infty \text { for all } q \in \mathbb{N}_{0}
$$

where the weight $(2 \mathbb{N})^{q \alpha}$ is defined as

$$
(2 \mathbb{N})^{q \alpha}=2^{q \alpha_{1}}(2 \cdot 2)^{q \alpha_{2}} \cdots .
$$

We equip $(S)$ with the projective topology based on the norms $\|\cdot\|_{0, q}, q \in \mathbb{N}_{0}$. The Hida distribution space is defined as the topological dual of $(S)$ and can be described as the collection of all formal chaos expansions $F(\omega)=\sum_{\alpha} a_{\alpha} \mathcal{H}_{\alpha}(\omega)$ such that there exists a $q \in \mathbb{N}_{0}$ with

$$
\|F\|_{0,-q}^{2}:=\sum_{\alpha} \alpha ! a_{\alpha}^{2}(2 \mathbb{N})^{-q \alpha}<\infty .
$$

We have the following chain of inclusions

$$
(S) \subset \mathcal{L}^{2}(\mu) \subset(S)^{*}
$$

The action of $F(\omega)=\sum_{\alpha} a_{\alpha} \mathcal{H}_{\alpha}(\omega) \in(S)^{*}$ on $f(\omega)=\sum_{\alpha} c_{\alpha} \mathcal{H}_{\alpha}(\omega) \in(S)$ can be represented by

$$
\langle F, f\rangle=\sum_{\alpha} \alpha ! a_{\alpha} c_{\alpha}
$$


A useful property of the Hida space $(S)^{*}$ is that it accomodates the white noise $W_{t}(\omega)$ of the Brownian motion $B_{t}(\omega)$, which is the time derivative of the Brownian motion (see [6]). The Hida distribution space $(S)^{*}$ can be endowed with structure of topological algebra with respect to the Wick product $\diamond$, which can be introduced as

$$
\mathcal{H}_{\alpha}(\omega) \diamond \mathcal{H}_{\beta}(\omega)=\mathcal{H}_{\alpha+\beta}(\omega) \text { for } \alpha, \beta \in \mathcal{I}
$$

The product is linearly extended to whole space $(S)^{*}$. In the sequel we will make use of the important tool of the Hermite transform, which is an algebra homomorphism between the Hida distribution space and the algebra of power series in infinitely many complex variables. The definition of the Hermite transform relies on the expansion along the basis $\left\{\mathcal{H}_{\alpha}\right\}_{\alpha \in \mathcal{I}}$. For $F(\omega)=\sum_{\alpha} c_{\alpha} \mathcal{H}_{\alpha}(\omega) \in(S)^{*}$ the Hermite transform of $F$, denoted by $(\mathcal{H} F)(z)$, is defined by

$$
(\mathcal{H} F)(z)=\sum_{\alpha} c_{\alpha} z^{\alpha} \in \mathbb{C}
$$

where $z=\left(z_{1}, z_{2}, \ldots\right) \in \mathbb{C}^{\mathbb{N}}$ ( the set of all complex valued sequences ) and $z^{\alpha}=z_{1}^{\alpha_{1}} z_{2}^{\alpha_{2}} \cdot$.. for $\alpha=\left(\alpha_{1}, \alpha_{2}, \ldots\right) \in \mathcal{I}$ with $z_{j}^{0}=1$, under the assumption that the series converges absolutely. One can prove that the Hermite transform (2.4) converges on the infinite dimensional neighbourhood $\mathbb{K}_{q}(R)$ in $\mathbb{C}^{\mathbb{N}}$ defined as

$$
\mathbb{K}_{q}(R)=\left\{\left(\xi_{1}, \xi_{2}, \ldots\right) \in \mathbb{C}^{\mathbb{N}}: \sum_{\alpha \neq 0}\left|\xi^{\alpha}\right|^{2}(2 \mathbb{N})^{q \alpha}<R^{2}\right\}
$$

for some $0<q, R<\infty$. It can be shown that the Hermite transform gives a unique characterization of any element in $(S)^{*}$ ( see [7],Theorem 2.6.11). The Hermite transform of the white noise takes the form

$$
\left(\mathcal{H} W_{t}\right)(z)=\sum_{k \geq 1} \xi_{k}(t) z_{k}
$$

Since $\mathcal{H}$ maps $(S)^{*}$ to the algebra of power series homomorphically, we find the identity

$$
\mathcal{H}(F \diamond G)(z)=\mathcal{H}(F)(z) \mathcal{H}(G)(z) .
$$

The latter relation (2.5) gives rise to an extension of the Wick version of complex analytical functions $g$ that can be expanded into a Taylor series around the point $\xi_{0}=\mathcal{H}(F)(0)$, with real valued coefficients. In virtue of the characterization theorem there exists a unique $Y \in(S)^{*}$ such that

$$
\mathcal{H}(Y)(z)=g(\mathcal{H}(F)(z))
$$

holds. We write $g^{\diamond}(F)=Y$ and call it the Wick version of $g$ applied to $F$. Using the Wick powers, defined by

$$
F^{\diamond n}=F \diamond F \diamond \cdots \diamond F(\text { n times) }
$$

we get for example the following representation of the Wick version of the exponential function:

$$
\exp \diamond(F)=\sum_{n \geq 0} \frac{1}{n} F^{\diamond n} .
$$




\section{Explicit solution of Itô-diffusions and its correspon- dence to the general stochastic transport equation}

Throughout the paper we consider the following Itô-diffusion

$$
d X_{t}=b\left(t, X_{t}\right) d t+\sigma\left(t, X_{t}\right) d B_{t}, X_{0}=x, 0 \leq t \leq T
$$

where the functions $b:[0, T] \times \mathbb{R} \rightarrow \mathbb{R}, \sigma:[0, T] \times \mathbb{R} \rightarrow \mathbb{R}$ are measurable and satisfy

$$
|b(t, x)|+|\sigma(t, x)| \leq C(1+|x|), x \in \mathbb{R}, t \in[0, T]
$$

for some constant $C$ and

$$
|b(t, x)-b(t, y)|+|\sigma(t, x)+\sigma(t, y)| \leq D|x-y|, x, y \in \mathbb{R}, t \in[0, T]
$$

for some constant $D$. It is well known that the above conditions entail the existence of strong solution $X_{t}$ such that

$$
E\left[\int_{0}^{T} X_{t}^{2} d t\right]<\infty .
$$

For notational convenience in the sequel we assume (3.1) to be time homogenous. In the one dimensional case the above conditions for strong solutions can be considerably relaxed by assuming for instance that $b$ is bounded and measurable and $\sigma$ Lipschitz continuous and bounded away from the origin on every compact subset of $\mathbb{R}$ ( see [11] for instance). Further we impose on $\sigma$ that

$$
\sigma(x)>0 \text { for all } x \in \mathbb{R} \text { and continuously differentiable }
$$

and we require

$$
\frac{b(x)}{\sigma(x)} \text { is bounded on } \mathbb{R} \text {. }
$$

For our main result we have to introduce a stochastic integral on the Hida space $(S)^{*}$ with respect to a 1 -dimensional Brownian motion $\hat{B}_{t}$ on the filtered probability space

$$
(\hat{\Omega}, \hat{\mathcal{F}}, \hat{\mu}),\left\{\hat{\mathcal{F}}_{t}\right\}_{t \geq 0}
$$

where $\hat{\mathcal{F}}_{t}$ is the $\hat{\mu}$-completed filtration generated by $\hat{B}_{t}$. We just give the construction with respect to the singular white noise $W_{s} \in(S)^{*}$ ( see Section 2 ). Our construction procedure indicates how the special case can be generalized to integration of predictable $(S)^{*}$-valued processes. For more information about stochastic integration on conuclear spaces in a general setting we refer to the book of Kallianpur and Xiong [13].

Choose a sequence of partitions $0=t_{1}^{n}<t_{2}^{n}<\cdots<t_{m_{n}}^{n}=T$ of the interval $[0, T]$ with $\max _{i}\left|t_{i+1}^{n}-t_{i}^{n}\right| \rightarrow 0$ for $n \rightarrow \infty$. Define

$$
W_{t}^{n}:=\sum_{i=1}^{m_{n}-1} 1_{\left[t_{i}^{n}, t_{i+1}^{n}[\right.}(t) W_{t_{i}^{n}}, t \in[0, T] .
$$

One observes that there exists a $q>1$ such that

$$
\left\|W_{t}^{n}-W_{t}\right\|_{-0,-q} \rightarrow 0 \text { as } n \rightarrow \infty .
$$


Let us define the stochastic integral of $W_{t}^{n}$ with respect to $\hat{B}_{t}$ as

$$
\int_{0}^{T} W_{s}^{n} d \hat{B}_{s}:=\sum_{i=1}^{m_{n}-1}\left(\hat{B}_{t_{i+1}^{n}}-\hat{B}_{t_{i}^{n}}\right) W_{t_{i}^{n}}
$$

Furthermore it can be verified that the following isometry holds

$$
E_{\hat{\mu}}\left\|\int_{0}^{T} W_{s}^{n} d \hat{B}_{s}-\int_{0}^{T} W_{s}^{m} d \hat{B}_{s}\right\|_{-0,-q}^{2}=\int_{0}^{T}\left\|W_{s}^{n}-W_{s}^{m}\right\|_{-0,-q}^{2} d s
$$

The latter relation motivates the construction of the stochastic integral of $W_{s}$ with respect to $\hat{B}_{s}$ denoted by

$$
\int_{0}^{T} W_{s} d \hat{B}_{s}
$$

as the unique element in $(S)^{*}(\hat{\mu}-$ a.e. $)$ given by

$$
\int_{0}^{T} W_{s} d \hat{B}_{s}:=\lim _{n \rightarrow \infty} \int_{0}^{T} W_{s}^{n} d \hat{B}_{s} \text { in }(S)^{*} .
$$

We now can state a Lemma which is essential for the proof of our result.

Lemma 3.1 The map

$$
\begin{gathered}
\Phi: \hat{\Omega} \rightarrow(S)^{*} \\
\hat{\omega} \mapsto \exp ^{\diamond}\left\{\int_{0}^{T} W_{s}(\omega) d \hat{B}_{s}(\hat{\omega})\right\}
\end{gathered}
$$

is Bochner integrable on the Hida space $(S)^{*}$ with respect to the measure $\hat{\mu}$.

Proof. For the proof of the Lemma it is sufficient to show that

$$
E_{\hat{\mu}}\left[\sup _{z \in \mathbb{K}_{q}(R)}\left|\mathcal{H}\left(\exp \diamond\left\{\int_{0}^{T} W_{s} d \hat{B}_{s}\right\}\right)\right|\right]<\infty
$$

for some $q$ and $R$ ( see definition in Section 2 ). This is due to the fact that one can find for a distribution $F \in(S)^{*}$ constants $C=C(q, R)>0$ and a number $r$ such that

$$
\|F\|_{-0,-r} \leq C \sup _{z \in \mathbb{K}_{q}(R)}|\mathcal{H}(F)|
$$

( see [7] ). On the other hand there exists a $l$ such that

$$
|\mathcal{H}(F)|(z) \leq\|F\|_{-0,-l} A(q, R) \leq \infty
$$

for all $z \in \mathbb{K}_{q}(R)$, where $A(q, R):=\left(\sum_{\alpha}(2 \mathbb{N})^{q \alpha}\left|z^{\alpha}\right|^{2}\right)^{\frac{1}{2}}$. Thus it follows from the last estimate that

$$
\begin{gathered}
E_{\hat{\mu}}\left[\sup _{z \in \mathbb{K}_{q}(R)}\left|\mathcal{H}\left(\exp \diamond\left\{\int_{0}^{T} W_{s} d \hat{B}_{s}\right\}\right)\right|\right] \leq \\
E_{\hat{\mu}}\left[\exp \sup _{z \in \mathbb{K}_{q}(R)}\left|\mathcal{H}\left(\int_{0}^{T} W_{s} d \hat{B}_{s}\right)\right|\right] \leq
\end{gathered}
$$




$$
\begin{gathered}
\left.E_{\hat{\mu}} \exp \left[A(q, R) \| \int_{0}^{T} W_{s} d \hat{B}_{s}\right) \|_{-0,-l}\right] \leq \\
\left.E_{\hat{\mu}}\left[1_{\left.\left\{\| \int_{0}^{T} W_{s} d \hat{B}_{s}\right) \|_{-0,-l} \leq 1\right\}} \exp \left[A(q, R) \| \int_{0}^{T} W_{s} d \hat{B}_{s}\right) \|_{-0,-l}\right]\right]+ \\
\left.+E_{\hat{\mu}} \exp \left[A(q, R) \| \int_{0}^{T} W_{s} d \hat{B}_{s}\right) \|_{-0,-l}^{2}\right] \\
=: \mathcal{I}_{1}+\mathcal{I}_{2} .
\end{gathered}
$$

According to our construction of the stochastic integral we have that

$$
\int_{0}^{T} W_{s} d \hat{B}_{s} \in(S)_{-l}^{*} \quad(\hat{\mu}-\text { a.e. })
$$

for $l$ large enough. Since $\int_{0}^{T} W_{s} d \hat{B}_{s}$ is a zero mean Gaussian random variable in $(S)_{-l}^{*}$ we can apply a well known result of Fernique ( see [4] ) and obtain that

$$
\mathcal{I}_{2}<\infty
$$

for $R$ sufficiently small. Since $\mathcal{I}_{1}<\infty$ the result follows.

Now we are coming to the main theorem:

Theorem 3.2 Assume that (3.2) and (3.3) hold. Define the strictly increasing function $\Lambda: \mathbb{R} \rightarrow \mathbb{R}$ given by

$$
\Lambda(y):=\left\{\begin{array}{cc}
\int_{x}^{y} \frac{1}{\sigma(u)} d u & , y>x \\
-\int_{y}^{x} \frac{1}{\sigma(u)} d u & , y \leq x
\end{array}\right.
$$

Further let $\varphi: \mathbb{R} \rightarrow \mathbb{R}$ be a measurable function and $\hat{B}_{t}$ the auxiliary Brownian motion such that

$$
\left(\varphi \circ \Lambda^{-1}\right)\left(\hat{B}_{t}\right) \in \mathcal{L}^{2}(\hat{\mu}) \text { for all } t \in[0, T]
$$

Then the strong solution $X_{t}^{x}$ in (3.1) takes the explicit form

$$
\varphi\left(X_{t}^{x}\right)=E_{\hat{\mu}}\left[\varphi\left(\Lambda^{-1}\left(\hat{B}_{t}\right)\right) M_{T}^{\diamond}\right]
$$

where

$$
\begin{aligned}
M_{T}^{\diamond} & =\exp ^{\diamond}\left[\int _ { 0 } ^ { T } \left(W_{s}-\frac{b\left(\Lambda^{-1}\left(\hat{B}_{s}\right)\right)}{\sigma\left(\Lambda^{-1}\left(\hat{B}_{s}\right)\right)}+\frac{1}{2} \sigma^{\prime}\left(\Lambda^{-1}\left(\hat{B}_{s}\right)\right) d \hat{B}_{s}-\right.\right. \\
& -\frac{1}{2} \int_{0}^{T}\left(W_{s}-\frac{b\left(\Lambda^{-1}\left(\hat{B}_{s}\right)\right)}{\sigma\left(\Lambda^{-1}\left(\hat{B}_{s}\right)\right)}+\frac{1}{2} \sigma^{\prime}\left(\Lambda^{-1}\left(\hat{B}_{s}\right)\right)^{\diamond 2} d s\right]
\end{aligned}
$$

Proof. Without loss of generality we give the proof of the theorem for the driftless case, i.e. we consider the diffusion

$$
d X_{t}^{x}=\sigma\left(X_{t}^{x}\right) d B_{t}, X_{0}^{x}=x
$$


First, we want to determine a certain expression for the Hermite transform of $\varphi\left(X_{t}^{x}\right)$. Let $z \in\left(\mathbb{C}^{\mathbb{N}}\right)_{c}$. Because of the relation between the Hermite transform and the $S$-transform ( see the proof of Theorem 2.7.10 in [7] ) we can write

$$
\begin{aligned}
& \mathcal{H}\left(\varphi\left(X_{t}^{x}\right)\right)(z)=E_{\mu} {\left[\varphi\left(X_{t}^{x}\right) \exp ^{\diamond}\left(\int_{\mathbb{R}} z_{1} \xi_{1}(s)+z_{2} \xi_{2}(s)+\cdots+z_{k} \xi_{k}(s) d B_{s}\right)\right]=} \\
&=E_{\mu}\left[\varphi\left(X_{t}^{x}\right) \exp ^{\diamond}\left(\int_{\mathbb{R}} \mathcal{H}\left(W_{s}\right)(z) d B_{s}\right)\right] .
\end{aligned}
$$

Then it follows from the Girsanov transform that

$$
\mathcal{H}\left(\varphi\left(X_{t}^{x}\right)\right)(z)=E_{\mu}\left[\varphi\left(\tilde{X}_{t}^{x}\right)\right]
$$

where $\tilde{X}_{t}^{x}(\omega)=X_{t}^{x}\left(\omega+\omega_{0}\right)$ with $\left\langle\omega_{0}, \cdot\right\rangle=\left(\mathcal{H}\left(W_{s}\right)(z), \cdot\right)_{\mathcal{L}^{2}(\mathbb{R})}$. Note that $\tilde{X}_{t}^{x}$ solves the diffusion

$$
d \tilde{X}_{t}^{x}=h(t) \sigma\left(\tilde{X}_{t}^{x}\right) d t+\sigma\left(\tilde{X}_{t}^{x}\right) d B_{t},
$$

where $h(t)=\mathcal{H}\left(W_{t}\right)(z)$. Further for $Z_{t}^{x}:=\Lambda\left(\tilde{X}_{t}^{x}\right)$, Itô's Lemma implies that

$$
d Z_{t}^{x}=h(t)-\frac{1}{2}\left(\sigma^{\prime} \circ \Lambda^{-1}\right)\left(Z_{t}^{x}\right) d t+d B_{t}, Z_{0}^{x}=\Lambda(x)
$$

Applying the Girsanov transform again we obtain that

$$
\mathcal{H}\left(\varphi\left(X_{t}^{x}\right)\right)(z)=E_{\mu}\left[\varphi\left(\tilde{X}_{t}^{x}\right)\right]=E_{\hat{\mu}}\left[\left(\varphi \circ \Lambda^{-1}\right)\left(\hat{B}_{t}\right) M_{T}\right]
$$

where

$$
\begin{aligned}
M_{T} & =\exp \left[\int_{0}^{T}\left(h(s)-\frac{1}{2}\left(\sigma^{\prime} \circ \Lambda^{-1}\right)\left(\hat{B}_{s}\right)\right) d \hat{B}_{s}-\right. \\
& \left.-\frac{1}{2} \int_{0}^{T}\left(h(s)-\frac{1}{2}\left(\sigma^{\prime} \circ \Lambda^{-1}\right)\left(\hat{B}_{s}\right)\right)^{2} d s\right]
\end{aligned}
$$

Using Lemma 3.1 we can apply the inverse Hermite transform to (3.6) and get

$$
\mathcal{H}\left(\varphi\left(X_{t}^{x}\right)\right)(z)=\mathcal{H}\left(E_{\hat{\mu}}\left[\left(\varphi \circ \Lambda^{-1}\right)\left(\hat{B}_{t}\right) M_{T}^{\diamond}\right]\right)(z)
$$

on $\mathbb{K}_{q}(R)$ for some $q, R$. The rest of the proof is an immediate consequence of the characterization theorem ( see Theorem 2.6.11 in [7] ).

Remark 3.3 (i) Since $\Lambda^{-1}\left(\hat{B}_{t}\right)$ solves an $S D E$ we see that $\Lambda^{-1}\left(\hat{B}_{t}\right) \in \mathcal{L}^{2}(\hat{\mu})$. So it follows from Theorem 3.2 that the strong solution (3.1) can be represented as

$$
X_{t}^{x}=E_{\hat{\mu}}\left[\Lambda^{-1}\left(\hat{B}_{t}\right) M_{T}^{\diamond}\right]
$$

(ii) Under certain conditions on the diffusion coefficient Theorem 3.1 can be generalized to the case of d-dimensional SDE's. It is also conceivable to relax the conditions on the diffusion coefficients by employing stopping time techniques. 
Our line of reasoning in Theorem 3.2 reveals an interesting relation between the Itô-diffusion and the generalized stochastic transport equation. More precisely relation (3.6) shows the correspondence of strong solutions between (3.1) and the following stochastic partial differential equation

$$
\left\{\begin{array}{rc}
\partial_{t} u(t, x, \omega)=\frac{1}{2} \sigma^{2}(x) \partial_{x x} u(t, x, \omega)+b(x) \partial_{x} u(t, x, \omega)+ & \\
+\sigma(x) W_{t, x}(\omega) \diamond \partial_{x} u(t, x, \omega)+K(t, x) \diamond u(t, x)+g(t, x) & (t, x) \in[0, T] \times \mathbb{R} \\
u(0, x)=f(x) & x \in \mathbb{R}
\end{array}\right.
$$

where $W_{t, x}$ is the (space-time) singular noise and $K(t, x), g(t, x)$ and $f(x)$ are given stochastic distributions. This type or similar versions of equation (3.9) were studied by several authors [19], [5], [13], [16].

Theorem 3.4 Consider the Cauchy problem for (3.9) in the following special case

$$
\left\{\begin{array}{rc}
\partial_{t} u(t, x, \omega)=\frac{1}{2} \sigma^{2}(x) \partial_{x x} u(t, x, \omega)+b(x) \partial_{x} u(t, x, \omega)+ & \\
+\sigma(x) W_{t}(\omega) \diamond \partial_{x} u(t, x, \omega) & (t, x) \in[0, T] \times \mathbb{R} \\
u(0, x)=\varphi(x) & x \in \mathbb{R}
\end{array}\right.
$$

where $\varphi: \mathbb{R} \rightarrow \mathbb{R}$ is such that $\left(\varphi \circ \Lambda^{-1}\right)\left(\hat{B}_{t}\right) \in \mathcal{L}^{2}(\hat{\mu})$. Then the strong solution $u(t, x, \omega)$ is given by

$$
u(t, x, \omega)=\varphi\left(X_{t}^{x}(\omega)\right) \in \mathcal{L}^{2}(\mu)
$$

where $X_{t}^{x}$ is the strong solution of (3.1). In particular, if $\varphi$ is the identity, we get the correspondence of $X_{t}^{x}$ to the following Wick-type stochastic flow equation:

$$
\partial_{t} X_{t}^{x}=\frac{1}{2} \sigma^{2}(x) \partial_{x x} X_{t}^{x}+b(x) \partial_{x} X_{t}^{x}+\sigma(x) W_{t}(\omega) \diamond \partial_{x} X_{t}^{x}
$$

Proof. Applying the Hermite transform and Feynman-Kac formula the theorem is proved by the relation (3.6).

Remark 3.5 The equation (3.9) can be regarded as a "stochastic Feynman-Kac formula", in the terminology of [15], since the averaging over the characteristic formula, i.e. the case $\varphi(x)=e^{i \lambda x}$, yields the classical Feynman-Kac formula.

Closely related results to Theorem 3.2 and 3.5 were attained by [15],[20]. In [15] the authors generalize the result of [20]; in their framework the Itô-diffusion is covered by a Cauchy problem for a second order linear parabolic stochastic partial differential equation driven by a cylindrical Brownian motion in a Hilbert space. Our approach is different from the latter authors' ones since our Cauchy problem involves the concept of Wick product. However in our context the cylindrical Brownian motion occurs in the solution formula in form of the stochastic integral of the singular white noise since the cylindrical Brownian motion in Hilbert spaces is uniquely related to Gaussian noise in conuclear spaces (see [13]). We believe that our approach leads to a natural reformulation of Ito diffusions in terms of the Cauchy problem (3.10). On the one hand we can derive formulas for strong solutions $X_{t}^{x}$ of Itô-diffusions in a form which gives insight into the structure of the process. It has also 
the advantage that the representation of $X_{t}^{x}$ is expressed in terms of the Wick product. In view of applications this representation is desirable since it enables Wick calculation and the use of the property of the Wick chain rule for Malliavin derivatives (see [3]). In smoothing the singular noise our method can be for instance applied to determine hedging formulas for portfolios based on the stock dynamic described by (3.1)( see the approach in [1] in the special case of a Black-Scholes market). We intend to give this application in a forthcoming paper. On the other hand the Cauchy problem (3.9) comprises other important equations like the Zakai equation(see [2]). Furthermore this method facilitates the extension to a non Gaussian proces as a driving noise ( see [10] ).

\section{Explicit representation of the Donsker delta function of an Itô-diffusion}

In this section we want to demonstrate how the results in the last section can be applied to derive an explicit formula for the Donsker delta function of the diffusion (3.1). From now on we restrict the diffusion process $X_{t}^{x}$ to belong to the Meyer-Watanabe space $\mathcal{D}$, i.e.

$$
X_{t}^{x} \in \mathcal{D}
$$

and we assume that the Malliavin variance of the process is non-degenerate, i.e.

$$
\omega \mapsto\left(\|\nabla X\|_{\mathcal{L}^{2}(\mathbb{R})}(\omega)\right)^{-1}
$$

is contained in $\bigcap_{p \geq 1} \mathcal{L}^{p}(\mu)$, where $\nabla$ denotes the infinite dimensional gradient ( see for precise definitions and notions [21]). For example if we require in (3.1) that $\sigma>0$ and $b$ are functions in $C^{\infty}(\mathbb{R})$ with all derivatives bounded then conditions (4.1) and (4.2) are fullfilled. The assumptions (4.1) and (4.2) imply the existence of the Donsker delta function $\delta \circ X_{t}^{x}$ of $X_{t}^{x}$ in the Watanabe distribution space $\mathcal{D}^{*}$ ( see [21]). Since the Hida test function space $(S)$ can be continuously embedded into $\mathcal{D}$, we obtain with the help of the results of the last section the following result.

Theorem 4.1 Suppose that the diffusion $X_{t}^{x}$ satisfies conditions (4.1) and (4.2). Then the Donsker delta function of $X_{t}^{x}$ exists in $(S)^{*}$ and is given by

$$
\begin{gathered}
\delta .\left(X_{t}^{x}\right): \mathbb{R} \rightarrow(S)^{*} \\
y \mapsto \frac{1}{2 \pi} \int_{\mathbb{R}} e^{-i \lambda y} E_{\hat{\mu}}\left[e^{-i \lambda \Lambda^{-1}\left(\hat{B}_{t}\right)} M_{T}^{\diamond}\right] d \lambda
\end{gathered}
$$

with $M_{T}^{\diamond}$ as in the Theorem 3.2.

Remark 4.2 The Donsker delta function of a diffusion was studied by a number of authors. See for instance [18], [12] and [21] to mention a few. However to the best of our knowledge no explicit formula of the Donsker delta function of a large class of diffusions driven by the Brownian motion was derived before. Similar results for Levy processes can be found in [14].

The explicit representation of the Donsker delta function of a diffusion is of theoretical importance as well as of considerable interest in applications. For example the Donsker delta function of a diffusion can be used to investigate local time of diffusions which has applications in many branches of probability theory. 


\section{Acknowledgments}

We thank F.E. Benth and B. Øksendal for helpful comments.

\section{References}

[1] Aase,K., Øksendal,B. and Ubøe,J.:"Using Donsker delta function to compute hedging strategies", Potential analysis, 14, 2001, pp. 351-374.

[2] Benth,F.E.:"A functional process solution to a stochastic partial differential equation with application to nonlinear filtering", Stochastics 51, 1994, pp. 195-216.

[3] Di Nunno,G., Øksendal,B. and Proske,F.:"White noise analysis for Levy processes", to appear in J.Funct.Anal., 2003.

[4] DaPrato,G. and Zabczyk,J.:"Stochastic equations in infinite dimensions", Cambridge University Press, Cambridge, 1992

[5] Gjessing,H.:"Wick calculus with applications to anticipating stochastic differential equations", Manuscript, University of Bergen, 1994

[6] Hida,T., Kuo,H.-H., Potthoff,J. and Streit,L.: "White noise analysis". Kluwer 1993.

[7] Holden,H.,Øksendal,B., Ubøe,J. and Zhang,T.-S.: "Stochastic Partial Differential Equations- A Modeling, White Noise Functional Approach" Birkhäuser, Boston 1996.

[8] Isobe,E. and Sato,Sh.:"Wiener-Hermite expansion of a process generated by an Itô stochastic differential equation", J.Appl.Prob, 20, 1993, pp. 754-765.

[9] Kunita,H.:"Stochastic differential equations and stochastic flows of diffeomorphisms", Ecole d'ete de Probabilites de SaintFlour,XII,1982,P.L.Hennenquin ed.,LNM 1097, Springer, Berlin, 1984,pp. 143-303.

[10] Kondratiev,Y., Da Silva,J.L, and Streit,L.:"Generalized Appell systems", Methods Funct. Analysis Topology, 3 (3), 1997, pp. 28-61.

[11] Karatzas,I. and Shreve,S.E.:"Brownian motion and stochastic calculus", second edition, Springer-Verlag, 1991.

[12] Kuo,H.H.:"White noise distribution theory", Prob and Stoch, Series, Boca Raton, FL: CRC Press, 1996.

[13] Kallianpur,G. and Xiong,J.:"Stochastic differential equations in infinite dimensional spaces", IMS Lecture NotesMonograph series, Vol 26, 1995.

[14] Mataramvura,S., Øksendal,B. and Proske,F.:"The Donsker delta function of a Levy process with application to chaos expansion of local time", Preprint series University of Oslo, 2003.

[15] Mikulevicius,R. and Rozovskii,B.:"Linear parabolic Stochastic PDE's and Wiener Chaos", SIAM J.Math.Anal. 29, 2, 1998 pp. $452-480$.

[16] Nualart,D. and Zakai,M.:"Generalized Brownian functionals and the solution to a stochastic partial differential equation", J.Func.Anal. 84,1989, pp. 279-296.

[17] Obata,N.:"White noise calculus and Fock space",LNM 1577, Berlin, Springer-Verlag, 1994.

[18] Potthoff,J. and Sundar,P.:"Law of large numbers and central limit theorem for Donsker's delta function of Diffusion", Potential Analysis, 5, 1996, pp. 487-504.

[19] Potthoff,J., Vage,G. and Watanabe H.:"Generalized solutions of linear parabolic stochastic partial differential equations", Appl.Math.Optim. 38, 1998, pp. 95-107.

[20] Veretennikov,A.Yu. and Krylov,N.V.:"On explicit formulas for solutions of stochastic differential equations", Math.USSR Sbornik, 29, 1976, pp. 229-256.

[21] Watanabe,S.:"Malliavin's calculus in terms of generalized wiener functionals", Theory and application of random fields, G.Kallianpur,ed., Springer Berlin, 1983. 